\title{
Determination of Plasma Protein-Bound Malondialdehyde by Derivative Spectrophotometry
}

\author{
Guillaume Lefêvre ${ }^{1}$, Christine Bonneau', Sophie Rahma ${ }^{1}$,Bernard Chanu ${ }^{2}$, Didier Brault ${ }^{1}$, Rémy Couderc $^{1}$ and \\ Jacqueline Etienne ${ }^{1}$
}

1 Service de Biochimie, Hôpital Tenon, Paris, France

2 Service de Médecine Interne et de Pathologie Vasculaire, Hôpital Saint Louis, Paris, France

Summary: We describe a method for the measurement of protein-bound malondialdehyde with the thiobarbituric acid reaction in human plasma using second-derivative spectrophotometry. Calibration was done by spectrum height measurement from the baseline at $532 \mathrm{~nm}$. The data were compared with those obtained by using conventional absorbance and fluorimetric measurements. The results were linear from 0.2 to $80 \mu \mathrm{mol} / 1$ and the detection limit was $0.19 \mu \mathrm{mol} / 1$. Within-run and between-run precision, evaluated by analysing pooled normal plasma, were 8 and $14 \%$ respectively. The method was tested for the influence of bilirubin, haemoglobin, glucose, urea, uric acid, sucrose and $\mathrm{N}$-acetyl-neuraminic acid which interfered in the colorimetric method but not in the technique proposed here. The mean $( \pm S D)$ malondialdehyde concentration determined in 59 healthy blood donors with the new assay was $0.34( \pm 0.14) \mu \mathrm{mol} / \mathrm{l}$. This assay procedure could represent an alternative to high-performance liquid chromatography for the measurement of malondialdehyde in biological media.

\section{Introduction}

Assay of malondialdehyde is the most commonly used test for evaluating lipid peroxidation (1). This measurement is based on the reaction of malondialdehyde with thiobarbituric acid to form a $1: 2$ adduct, which has a stable pink colour that absorbs maximally at $532 \mathrm{~nm}$ or is fluorescent at $553 \mathrm{~nm}$, usually after extraction into $n$ butanol $(1-3)$. The use of the thiobarbituric acid reaction to determine lipid peroxidation in complex biological media such as serum and plasma is limited by its lack of specificity, which can be partly overcome by modifying the reaction conditions and/or improving the detection system. Many different substances, including lipid-derived monofunctional aldehydes, sucrose and ethanol, readily form adducts with thiobarbituric acid which contribute to the reaction $(1,4,5)$. The derivative spectrophotometry assay can be used to analyse mixtures of chromophores. Its efficiency is a function of the relative position of the absorbance maxima, and increases with the order of the derivative. Given its high discriminatory power, derivative spectrophotometry overcomes interference by compounds with a broader spectrum, and its specificity is better than that of conventional analytical spectrophotometry (6).

With the exception of the method described by Espinosa-Mansilla et al., derivative spectrophotometry has not been applied to the measurement of malondialdehyde in biological media (7). In this study, we quantified protein-bound malondialdehyde in plasma by means of second-derivative spectrophotometry and tested the in- fluence of various interfering substances commonly found in plasma.

\section{Materials and Methods \\ Apparatus}

A Uvikon 860 spectrophotometer with a Plotter 800 chart recorder (Kontron Instrument, Montigny le Bretonneux, France) was used for absorbance measurements. Fluorescence was measured on a Perkin-Elmer MPF-3 spectrofluorometer (Perkin-Elmer Instrument, Montigny le Bretonneux, France).

\section{Reagents and standards}

Thiobarbituric acid, $n$-butanol (fluorometric grade) and 1,1,3,3tetraethoxypropane were from Sigma Chemical Co (via Coger, France), and other reagents were from Prolabo (Paris, France). They were used without further purification. The control serum was lyophilised Biotrol OS (BioMérieux, Lyon, France) rehydrated daily according to the manufacturer's instructions.

\section{Samples}

Plasma was isolated from blood collected on EDTA tripotassium salt (Beckton Dickinson Vacutainer Systems, Grenoble, France) and centrifuged within $30 \mathrm{~min}$. The samples were kept at $4^{\circ} \mathrm{C}$ and used within 24 hours.

\section{Procedure for determining malondialdehyde}

Plasma protein bound malondialdehyde was determined according to Satoh (3), with the following modifications. Plasma was prepared by centrifugation of whole blood collected on EDTA. Each determination was performed in duplicate. Protein was precipitated by adding $2.5 \mathrm{ml}$ of $200 \mathrm{~g} / \mathrm{l}$ trichloroacetic acid to $0.5 \mathrm{ml}$ of plasma. After centrifugation at $1000 \mathrm{~g}$ for $10 \mathrm{~min}$, the supernatant was drawn off and the pellet rinsed with $2.5 \mathrm{ml}$ of acetic acid $(100 \mathrm{ml} / \mathrm{l}$ in distilled water). The protein precipitate was resuspended in 2.5 $\mathrm{ml}$ of acetic acid, and $3 \mathrm{ml}$ of thiobarbituric acid $(2 \mathrm{~g} / \mathrm{l}$ in $2 \mathrm{~mol} / \mathrm{l}$ 
$\mathrm{Na}_{2} \mathrm{SO}_{4}$ ) was added. The reaction mixture was heated in a boiling water bath for $30 \mathrm{~min}$, then rapidly cooled in an ice bath to stop the reaction. The chromophore was extracted in $4 \mathrm{ml}$ of $n$-butanol, and the organic supernatant isolated by centrifugation for $10 \mathrm{~min}$ at $3000 \mathrm{~g}$.

Fluorescence and absorbance measurements were performed on $n$ butanol extracts. Absorbance measurements were carried out at a wavelength of $532 \mathrm{~nm}$, with $n$-butanol as the blank in the reference channel. Derivative spectrophotometry measurements were made by recording the spectrum of the extract from 400 to $700 \mathrm{~nm}$ using $n$-butanol as the blank in the reference channel. The scale expansions for the second-derivative spectrum were selected between +0.001 and -0.001 units of absorbance per $\mathrm{nm}^{2}$, full scale. The baseline-measurements method was used at $532 \mathrm{~nm}$ for quantitation (6). Fluorimetric measurements were made at $515 \mathrm{~nm}$ $\left(\lambda_{\text {excitation }}\right)$ and $553 \mathrm{~nm}\left(\lambda_{\text {emission }}\right)$. The sample sensitivity was set at 10 , and full scale $(100 \%)$ was determined from the fluorescence intensity obtained with the $10 \mu \mathrm{mol} / 1$ 1,1,3,3-tetraethoxypropane calibration point.

Calibration was done with a stock solution of 1,1,3,3-tetraethoxypropane (1.1 g 1,1,3,3-tetraethoxypropane in $100 \mathrm{ml}$ acetic acid, $100 \mathrm{ml} / \mathrm{l})$. Appropriate dilutions were made to obtain concentrations equivalent to $0.1-10 \mu \mathrm{mol} / \mathrm{l} 1,1,3,3$-tetraethoxypropane. Each calibration point was determined in duplicate. The samples were heated in a boiling water bath for $30 \mathrm{~min}$. After extraction with n-butanol, absorbance or fluorescence were measured as specified for plasma samples.

The influence of haemoglobin and bilirubin was studied by spiking pooled normal human EDTA plasma with either a haemolysate or bilirubin (Lee Scientific Inc., via Coger, France) (8). Five standard reference materials (glucose, urea, uric acid, sucrose, $\mathrm{N}$-acetyl-neuraminic acid, all purchased from Sigma were added to EDTA plasma in order to test their influence on the malondialdehyde de-. termination. Plasma protein bound malondialdehyde was also determined in 56 healthy subjects and 19 hyperlipoproteinaemic subjects (9 with type IIa and 10 with type IIb according to Fredrickson) who gave informed consent to the study, and 42 patients presenting with jaundice.

Statistical evaluation of the data was carried out on a computer (Apple Macintosh), using Statview II software (Abacus Concept ${ }^{\circledR}$ ). Student t-test was used to estimate differences between data from patients and controls. The threshold of statistical significance was $\mathrm{p}<0.05$

\section{Results}

Calibration was carried out by constructing a standard curve for the second derivative at $532 \mathrm{~nm}$ with 9 samples containing a 1,1,3,3-tetraethoxypropane standard at concentrations ranging from 0 to $10 \mu \mathrm{mol} / \mathrm{l}$. The regression equation and the correlation coefficient were as follows: $y=5.982 x+0.791(y=$ signal height at $432 \mathrm{~nm}$ (in $\mathrm{mm}$ ); $\mathrm{x}=1,1,3,3$-tetraethoxypropane in $\mu \mathrm{mol} / \mathrm{l}$ ), $\mathrm{r}=0.998$.

The detection limit was $0.19 \mu \mathrm{mol} / \mathrm{l}$. Inter-assay precision of the calibration curves was determined $(\mathrm{n}=34)$ on six 1,1,3,3-tetraethoxypropane samples containing $0.25,0.50,1.00,2.50,5.00$ and $10.00 \mu \mathrm{mol} / \mathrm{l}$ 1,1,3,3-tetraethoxypropane. The height values (mean $\pm \mathrm{SD}$ ) expressed in $\mathrm{mm}$ were $2.28 \pm 0.29,4.01 \pm 0.36$, $7.00 \pm 0.37,15.86 \pm 0.41,30.89 \pm 0.47,60.48 \pm 0.61$ with CVs of $7.8 \%, 8.9 \%, 5.3 \%, 2.6 \%, 1.5 \%$ and $1.0 \%$ respectively. Linearity was tested in the range of 0 to $100 \mu \mathrm{mol} / \mathrm{l}$ using the baseline measurement at $532 \mathrm{~nm}$.
The assay remained linear up to at least $50 \mu \mathrm{mol} / \mathrm{l}$ of 1,1,3,3-tetraethoxypropane $(r=0.999, p<0.0002)$. Inter-assay precision was determined by analysing the Biotrol OS control in each run. A mean value of 1.70 $(\mathrm{SD}=0.11) \mu \mathrm{mol} / \mathrm{l}$ and a CV of $6 \%$ (range $1.52-1.96$ $\mu \mathrm{mol} / \mathrm{l})$ was found. Measurements of 1,1,3,3-tetraethoxypropane standard samples $(n=29)$ gave a run-torun coefficient of variation for the slopes of the calibration curve of $2.2 \%$. Within-run, and between-run precision, evaluated by analysing pooled normal plasma, was 8 and $14 \%$ respectively. Values of protein-bound malondialdehyde obtained by the proposed method ( $x$ ) and fluorometric assay (y) were strongly correlated: $\mathrm{y}=-0.031+1.179 \mathrm{x} ; \mathrm{r}=0.991 ; \mathrm{p}<0.001 ; \mathrm{n}=35$. Values determined in 56 healthy subjects with the proposed method were $0.33 \pm 0.14 \mu \mathrm{mol} / \mathrm{l}$ (range $0.12-$ $0.78 \mu \mathrm{mol} / \mathrm{l})$, and with fluorometric assay $1.03 \pm 0.11$ (range $0.84-1.00 \mu \mathrm{mol} / \mathrm{l}$ ).

Bilirubin and haemoglobin interferences were studied by spiking plasma with increasing concentrations. As shown in figure 1 , a single absorbance measurement carried out at $532 \mathrm{~nm}$ yielded falsely enhanced proteinbound malondialdehyde concentrations. The use of second derivative spectrophotometry cancelled these influences, since plasma-, bilirubin- and haemoglobin-spiked plasma exhibited the same second-derivative spectra

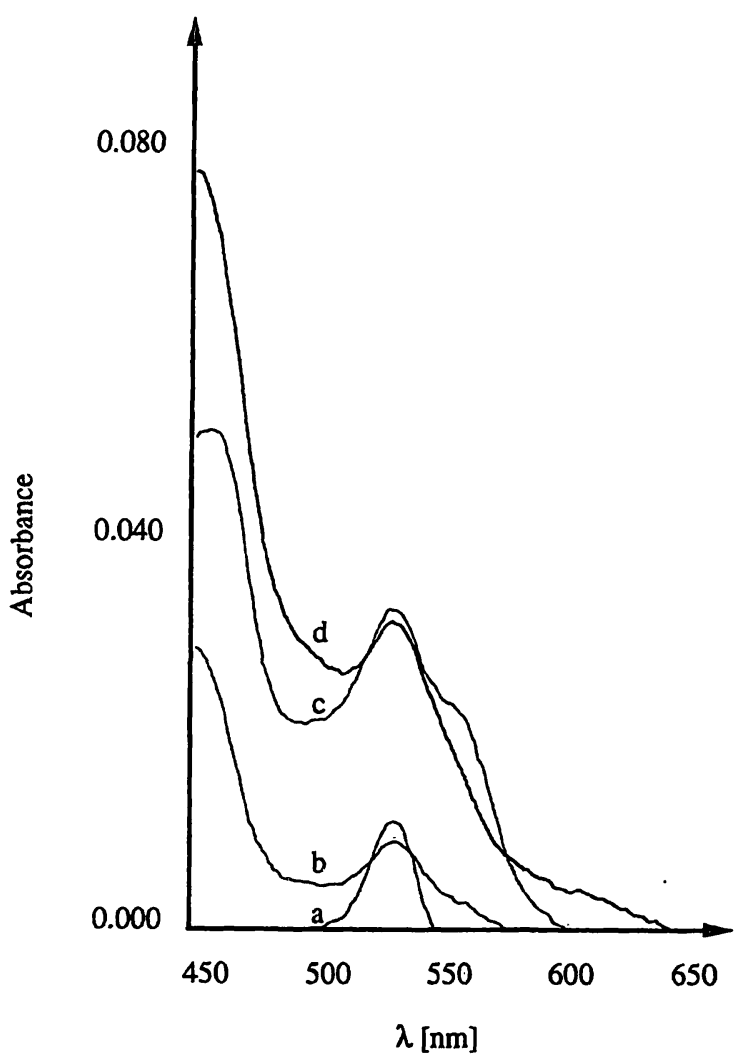

Fig. 1 Conventional spectra of malondialdehyde $(0.30 \mu \mathrm{mol} / \mathrm{l})$ (a), unspiked plasma (malondialdehyde concentration $=0.50$ $\mu \mathrm{mol} / \mathrm{l}$ ) (b), haemoglobin-spiked plasma (haemoglobin concentration $=125 \mu \mathrm{mol} / \mathrm{l})(\mathrm{c})$ and bilirubin-spiked plasma (total bilirubin concentration $=50 \mu \mathrm{mol} / \mathrm{l})(\mathrm{d})$ obtained after reaction with thiobarbituric acid. 


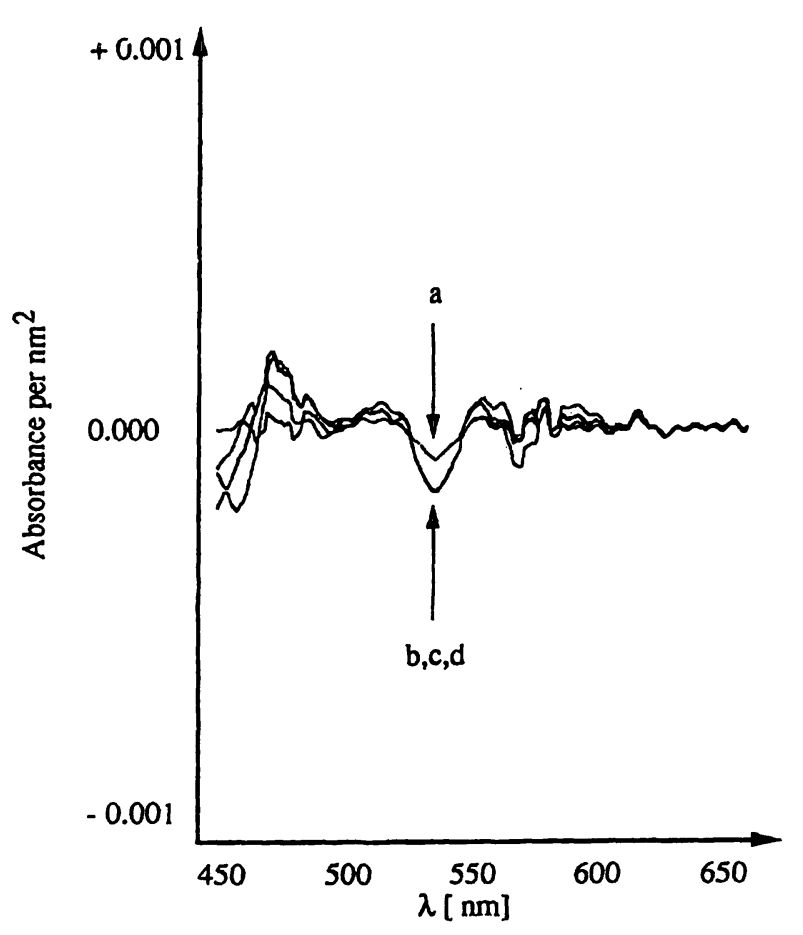

Fig. 2 Second derivative spectra of malondialdehyde $(0.30$ $\mu \mathrm{mol} / \mathrm{l}$ ) (a), unspiked plasma (malondialdehyde concentration $=0.50 \mu \mathrm{mol} / \mathrm{l}$ ) (b), haemoglobin-spiked plasma (haemoglobin concentration $=125 \mu \mathrm{mol} / \mathrm{l})(\mathrm{c})$ and bilirubin-spiked plasma (total bilirubin concentration $=50 \mu \mathrm{mol} / \mathrm{l})(\mathrm{d})$ obtained after reaction with thiobarbituric acid.

(fig. 2). No net influence was found when measurements were made with fluorescence or derivative spectrophotometry, even when high bilirubin or haemoglobin concentrations were used. In contrast, absorbance measurements at a single wavelength showed false positive interference by bilirubin or haemoglobin which increased with concentration (fig. 3 ).

These results were confirmed by determining the apparent plasma protein-bound malondialdehyde concentration by the absorbance method and the proposed method in 52 samples from 42 patients with jaundice. Figure 4 shows the relation between the total bilirubin concentra-

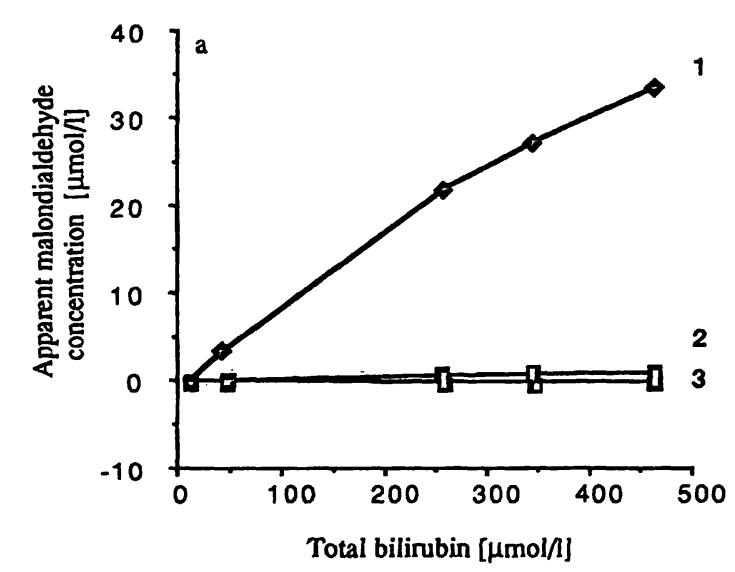

Fig. 3 Influence of bilirubin (a) and haemoglobin (b) on the apparent malondialdehyde concentration. Data are means of three experiments. The apparent malondialdehyde concentration $(\mu \mathrm{mol} / \mathrm{l})$ was calculated as the difference between the values of each spiked sample and that obtained without spiking: tion and the apparent plasma protein-bound malondialdehyde concentration. Total bilirubin $(x)$ and malondialdehyde values did not correlate in the derivative spectrophotometry method $(n=52 ; r=0.071 ; p=0.61)$, whereas a strong correlation existed when absorbance was only measured at $532 \mathrm{~nm}$ : $\mathrm{y}=1.3473+0.0574 \mathrm{x}$; $\mathrm{n}=52 ; \mathrm{r}=0.877 ; \mathrm{p}<0.001$.

Plasma spiked with glucose, sucrose, urea, uric acid and $\mathrm{N}$-acetyl-neuraminic acid at the final concentrations listed in the table 1, had no influence on malondialdehyde concentrations calculated from derivative spectrophotometric values.

The proposed method was applied to the study of lipid peroxidation in patients with type II hyperlipoproteinaemia (tab. 2). A significant increase of protein bound malondialdehyde was found in hyperlipoproteinaemia type IIb patients. No influence of sex or age on protein bound malondialdehyde levels could be demonstrated. Protein bound malondialdehyde determined with the proposed method was correlated neither with lipid (total cholesterol, triacylglycerols) nor apolipoprotein concentrations (apolipoprotein A-I, apolipoprotein B).

\section{Discussion}

Malondialdehyde assay after reaction with thiobarbituric acid is the most commonly used method for assessing lipid peroxidation in biological samples. Apparent malondialdehyde concentrations determined in complex media such as plasma have three different origins. The first is malondialdehyde already present in the sample. The second is aldehydes, which can react with thiobarbituric acid to give so-called "thiobarbituric acid reactant substances". These compounds originate from either biological markers of lipid peroxidation or oxidation of polyunsaturated fatty acids in vitro. The third is biological substances and xenobiotics that can interfere with

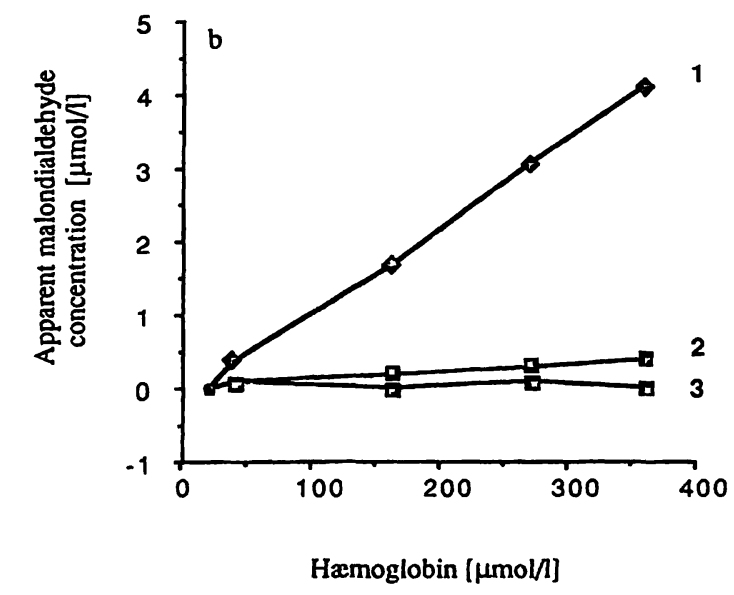

1: singlc absorbance determination at $532 \mathrm{~nm}$.

2: fluorometric determination.

3: derivative spectrophotometry determination. 
thiobarbituric acid assays (1). We propose here an assay of protein-bound malondialdehyde which avoids most interferences by using derivative spectrophotometry.

Accurate malondialdehyde assay must avoid polyunsaturated fatty acids oxidation and malondialdehyde formation in vitro. Since the clot formation and the presence

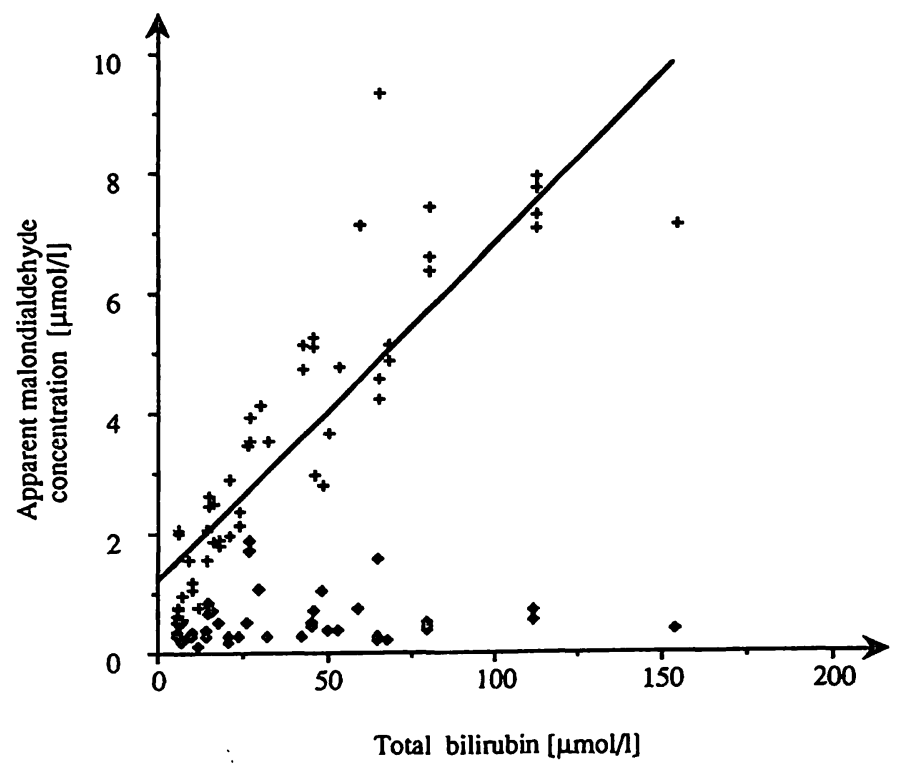

Fig. 4 Values of malondialdehyde in subjects with jaundice. Correlations between total bilirubin concentration $(x)$ and apparent malondialdehyde concentrations (y) calculated from absorbance at 532 $\mathrm{nm}(+)$ and derivative spectrophotometry $(\diamond)$. Total bilirubin and apparent malondialdehyde values did not correlate in derivative spectrophotometry, whereas there was a significant correlation in single absorbance measurements.

Tab. 1 Test for interference in the derivative spectrophotometric assay for plasma malondialdehyde. Plasma samples were spiked with the indicated concentrations.

\begin{tabular}{lc}
\hline Substances & Concentration added per litre \\
\hline Urea & $32 \mu \mathrm{mmol} / 1$ \\
Glucose & $20 \mathrm{mmol} / 1$ \\
Sucrose & $10 \mathrm{mmol} / 1$ \\
Uric acid & $1000 \mu \mathrm{mol} / 1$ \\
N-Acetyl-neuraminic acid & $500 \mu \mathrm{mol} / 1$ \\
\hline
\end{tabular}

of metal ions favours malondialdehyde formation, collection of blood on anticoagulants and on chelators is mandatory (9-12). According to Carbonneau et al. (13), malondialdehyde in plasma is mostly bound to proteins (83\%), a result consistent with those of Hackett et al. (14) and Lepage et al. (15) who found a low recovery from free malondialdehyde added to plasma, and those of Largillière \& Mélançon who were unable to find free malondialdehyde within plasma samples using a HPLC method (16).

In the proposed method, the malondialdehyde-thiobarbituric acid adduct is determined in the infranatant obtained after precipitation of protein, and absorbances are measured on the $n$-butanol extract. The use of acidic $\mathrm{pH}$ allows protein precipitation and elimination of watersoluble interferents, as well as the thiobarbituric acid reaction with aldehydes which is completed at a $\mathrm{pH}$ near $3(3,17)$. We were unable to realize the malondialdehyde assay without previous precipitation of plasma or without using acidic $\mathrm{pH}$ (data not shown).

We confirmed, as previously reported by Satoh, that precipitation with trichloroacetic acid significantly diminished influences from water-soluble interferents including $\mathrm{N}$-acetyl-neuraminic acid (3), but not haemoglobin and albumin-bound bilirubin which are found almost entirely in the acidic precipitate. When the plasma haemoglobin concentration is increased to $175 \mathrm{mg} / \mathrm{l}$ by the addition of a haemolysate, the apparent lipoperoxide concentration is increased 2- to 4-fold, a result consistent with the data reported here (18). Furthermore, since patients with liver failure are often jaundiced, the determination of plasma lipid peroxides needs to be free from bilirubin interference $(19-21)$.

With conventional absorbance measurements at a single wavelength, haemoglobin and bilirubin interfere with the malondialdehyde determination because the spectra overlap. Derivative spectrophotometry separates peaks since the absorbance maxima of haemoglobin and bilirubin are different from that of malondialdehyde after re-

Tab. 2 Plasma lipid, apolipoprotein and protein-bound malondialdehyde levels in patients with type Ila and Ilb hyperlipoproteinaemia.

\begin{tabular}{|c|c|c|c|}
\hline \multirow[t]{2}{*}{ Quantities } & \multirow{2}{*}{$\begin{array}{l}\text { Controls } \\
(n=11)\end{array}$} & \multicolumn{2}{|l|}{ Patients } \\
\hline & & $\begin{array}{l}\text { Hyperlipoproteinaemia } \\
\text { type IIa } \\
(\mathrm{n}=9)\end{array}$ & $\begin{array}{l}\text { Hyperlipoproteinaemia } \\
\text { type IIb } \\
(\mathrm{n}=10)\end{array}$ \\
\hline Total cholesterol $(\mathrm{mmol} / \mathrm{l})$ & $4.67 \pm 0.49$ & $10.06 \pm 2.41^{\mathrm{c}}$ & $7.56 \pm 1.13^{c}$ \\
\hline Triacylglycerols (mmol/l) & $0.78 \pm 0.26$ & $1.03 \pm 0.41$ & $3.16 \pm 0.98^{c}$ \\
\hline Apolipoprotein A-I $(g / 1)$ & $1.34 \pm 0.16$ & $1.35 \pm 0.28$ & $1.31 \pm 0.29$ \\
\hline Apolipoprotein B (g/l) & $1.00 \pm 0.20$ & $2.26 \pm 0.62^{c}$ & $1.65 \pm 0.50^{\mathrm{b}}$ \\
\hline Apolipoprotein C-II (mg/l) & $34 \pm 10$ & $49 \pm 17$ & $102 \pm 21^{c}$ \\
\hline Apolipoprotein C-III (mg/l) & $84 \pm 22$ & $120 \pm 26^{\mathrm{a}}$ & $260 \pm 80^{c}$ \\
\hline Apolipoprotein E (mg/l) & $42 \pm 8$ & $71 \pm 21^{b}$ & $92 \pm 33^{b}$ \\
\hline Protein-bound malondialdehyde $(\mu \mathrm{mol} / \mathrm{l})$ & $0.27 \pm 0.12$ & $0.35 \pm 0.22$ & $0.56 \pm 0.22^{\mathrm{a}}$ \\
\hline
\end{tabular}

Significantly different from controls: ${ }^{\mathrm{a}} \mathrm{p}<0.05 ;{ }^{\mathrm{b}} \mathrm{p}<0.01 ;{ }^{\mathrm{c}} \mathrm{p}<0.001$. 
action with thiobarbituric acid. This is also true for the other compounds listed in the table 1. Application of derivative spectrophotometry to plasma malondialdehyde measurement was first published by EspinosaMansilla et al. who used acetonitrile to precipitate plasma proteins and used the first-derivative of the absorbance spectrum for quantification (7). The present report confirms these results and demonstrates that protein-bound interferents in the thiobarbituric acid test can be eliminated by derivative spectrophotometry.

Oxidative stress occurs in various human diseases, and modifications of low-density lipoprotein by free radicals are supposed to play a role in atherogenesis (22). However, conflicting blood concentrations of malondialdehyde or thiobarbituric acid reactant substances in patients with high prevalence of atherosclerosis have been reported $(9,23)$. We demonstrated in this study that protein-bound malondialdehyde concentrations were increased in patients with type Ilb hypercholesterolaemia. Different authors have found significant correlations between lipid and thiobarbituric acid reactant substances levels. For example, Hoving et al., using an HPLC method for determining blood malondialdehyde concentration, demonstrated a relationship between malondialdehyde and lipids (e.g.: triacylglycerol and cholesterol levels) respectively, whereas Öhrvall et al. suggested that blood fatty acid composition influences malondialdehyde levels $(24,25)$. In our study, no correlation could be found either with normal or hyperlipoproteinaemic patients, a result consistent with those of Plachta et al. who failed to demonstrate a significant correlation between lipid and lipid peroxide levels, determined by Yagi's method (23). More results are needed before suggesting that the derivative spectro-

\section{References}

1. Janero DR. Malondialdehyde and thiobarbituric acid reactivity as diagnostic indices lipid peroxidation and peroxidative tissue injury. Free Rad Biol Med 1990; 9:515-40.

2. Yagi K. A simple fluorometric assay for lipoperoxides in blood plasma. Biochem Med 1976; 15:212-6.

3. Satoh K. Serum lipid peroxides in cerebrovascular disorders determinated by a new colorimetric method. Clin Chim Acta 1978; 90:37-43.

4. Kosugi $H$, Kojima T, Kikugawa $K$. Thiobarbituric acid reactive substances from peroxidized lipids. Lipids $1989 ; 24: 873-81$.

5. Gutteridge JMC, Tickner TR. The thiobarbituric acid-reactivity of bile pigments. Biochem Med 1978; 19:127-32.

6. O'Haver TC. Potential clinical applications of derivative and wavelength modulation spectrometry. Clin Chem 1979; 25:1548-53.

7. Espinosa-Mansilla A, Salinas F, Leal AR. Determination of malondialdehyde in human plasma: elimination of spectral interferences in the 2-thiobarbituric acid reaction. Analyst 1993; 118:89-95.

8. Commission for "Validation of methods" of the Société Française de Biologie Clinique Appendix 3 and 9. Ann Biol Clin 1986; 44:719-45.

9. Kameda-Takemura K, Corder CN, Lee DM. Lipid peroxide levels in type II hyperlipoproteinemic subjects. Artery 1993; 20:189-200. photometric method is more specific than classical thiobarbituric acid reactant substances tests, on the basis that avoids the influence of plasma lipid on malondialdehyde levels.

The measured mean lipid peroxide concentrations in the plasma of healthy subjects are lower when HPLC techniques are used (reviewed 1.c. (26)). This is due to the separation of thiobarbituric acid-malondialdehyde adducts from contaminants which absorb or fluorescence at the same wavelengths as malondialdehyde. Reported malondialdehyde concentrations in blood range from 0.6 to $4.9 \mu \mathrm{mol} / 1$, but values are far lower with HPLC methods $(12,27,28)$. The malondialdehyde protein-bound values found in this study were also very low, in the range of HPLC values, suggesting that the derivative spectrophotometry assay may be as specific as HPLC.

\section{Conclusion}

Finally, our results also confirm that malondialdehyde cannot properly be measured in plasma by classical spectrophotometric methods. The proposed procedure represents an alternative to HPLC as proposed by Espinosa-Mansilla et al. (7) and can be used to measure protein-bound malondialdehyde in plasma samples containing high concentrations of bilirubin, haemoglobin and other interfering substances.

\section{Acknowledgements}

The authors wish to thank Dr J. Maury (Banque du Sang, Hôpital Tenon) for providing samples from blood donors and Virginie $\mathrm{Ba}$ rathon for typing the manuscript.

10. Wade CR, Van Rij AM. Plasma thiobarbituric acid reactivity: reaction conditions and the role of iron, antioxidants and lipid peroxy radicals on the quantitation of plasma lipid peroxides. Life Sci 1988; 43:1085-93.

11. Bigwood T, Read G. Pseudomalondialdehyde activity in the thiobarbituric acid test. Free Rad Res Communs 1989; 6:387-92.

12. Wong SHY, Knight JA, Hopfer SM, Zaharla O, Leach Jr CN, Sunderman FW. Lipoperoxides in plasma as measured by liquid-chromatographic separation of malondialdehyde-thiobarbituric acid adduct. Clin Chem 1987; 33:214-20.

13. Carbonneau MA, Peuchant E, Sess D, Canioni P, Clerc M. Free and bound malondialdehyde measured as thiobarbituric acid adduct by HPLC in serum and plasma. Clin Chem $1991 ; 37: 1423-9$.

14. Hackett C, Linley-Adams L, Loyd B, Walker V. Plasma malondialdehyde: a poor measure of in vivo lipid peroxidation. Clin Chem 1988; 34:208.

15. Lepage G, Munoz G, Champagne J, Roy CC. Preparative steps necessary for the accurate measurement of malondialdehyde by HPLC. Anal Biochem 1991; 197:277-83.

16. Larguillière $C$, Mélançon $S B$. Free malondialdehyde determination in human plasma by high-performance liquid chromatography. Anal Biochem 1988; 170:123-6. 
17. Bird RP, Draper HH. Comparative studies on different methods of malondialdehyde determination. Meth Enzymol 1984; 105:299-305.

18. Gilbert HS. Stump DD, Roth EF Jr. A method to correct for errors caused by generation of interfering compounds during erythrocyte lipid peroxidation. Anal Biochem 1984; 137:282-6.

19. Yagi K. Lipid peroxides and human diseases. Chem Phys Lipids 1987; 45:337-51.

20. Tsai LY, Lee KT, Tsai SM, Lee SC, Yu HS. Changes of lipid peroxide levels in blood and liver tissue of patients with obstructive jaundice. Clin Chim Acta 1993; 215:41-50.

21. Lemonnier F, Cresteil D, Féneant $M$, Couturier $M$, Bernard $O$, Alagille D. Plasma lipid peroxides in cholestatic children. Acta Paediatr Scand 1987; 76:928-34.

22. Witzum JL, Steinberg D. Role of oxidized low-density lipoprotein in atherogenesis. J Clin Invest 1991; 88:1785-92.

23. Plachta $\mathrm{H}$, Bartnikowska $\mathrm{C}$, Obara A. Lipid peroxides in blood from patients with atherosclerosis of coronary and peripheral arteries. Clin Chim Acta 1992; 211:101-12.

24. Hoving EB, Laing C, Rutgers HM, Teggeler M, Van Doormaal JJ, Juskiet FAJ. Optimized determination of malondialdehyde in plasma lipid extracts using 1,3-diethyl-2-thiobarbituric acid: influence of detection method and relations with lipids and fatty acids in plasma from healthy adults. Clin Chim Acta 1992; 208:63-76.

25. Öhrvall M, Tengblad S, Ekstrand B, Siebbahn A, Vessby B. Malondialdehyde concentration in plasma is inversely correlated to the proportion of linoleic acid in serum lipoprotein lipids. Atherosclerosis 1994; 108:103-10.

26. Wade CR, Van Rij AM. Plasma malondialdehyde, lipid peroxides and the thiobarbituric acid reaction. Clin Chem 1989; 35:336.

27. Tatum VL, Changhitb C, Chow CK. Measurements of malondialdehyde by high performance liquid chromatography with fluorescence detection. Lipids 1900; 25:226-9.

28. Young LS, Trimble ER. Measurement of malondialdehyde in plasma by high performance liquid chromatography with fluorimetric detection. Ann Clin Biochem 1991; 28:504-8.

\section{Received March 25, 1996}

Corresponding author: Dr G. Lefèvre, Service de Biochimie, Hôpital Tenon, 4, Rue de la Chine, F-75970 Paris Cedex 20, France 